\title{
EIGENVALUES AND RANGES FOR PERTURBATIONS OF NONLINEAR ACCRETIVE AND MONOTONE OPERATORS IN BANACH SPACES
}

\author{
ZHOU HAIYUN AND ATHANASSIOS G. KARTSATOS
}

\begin{abstract}
Various eigenvalue and range results are given for perturbations of $m$-accretive and maximal monotone operators. The eigenvalue results improve and extend some recent results by Guan and Kartsatos, while the range theorem gives an affirmative answer to a recent problem of Kartsatos.
\end{abstract}

\section{INTRODUCTION AND PRELIMINARIES}

Let $X$ be a real Banach space with norm $\|\cdot\|$ and normalized duality mapping $J$. An operator $T: X \supset D(T) \rightarrow 2^{X}$ is said to be "accretive" if for every $x, y \in D(T)$ and every $u \in T x, v \in T y$ there exists $j \in J(x-y)$ such that

$$
<u-v, j>\geq 0
$$

An accretive operator $T$ is "strongly accretive" if there exists a positive constant $c$ such that the inequality (1) holds with 0 replaced by $c\|x-y\|^{2}$. An accretive operator $T$ is " $m$-accretive" if $R(T+\lambda I)=X$ for some $\lambda>0$, (or, equivalently, for all $\lambda>0$ ). For an $m$-accretive operator $T$, the resolvents $J_{\lambda}: X \rightarrow D(T)$ of $T$ are defined by $J_{\lambda}=(I+\lambda T)^{-1}$ for all $\lambda>0$. The Yosida approximants $T_{\lambda}: X \rightarrow X$ of $T$ are defined by $T_{\lambda}=\frac{1}{\lambda}\left(I-J_{\lambda}\right)$. For the main properties of $J_{\lambda}$ and $T_{\lambda}$ the reader is referred to Barbu [1], Browder[2] and Lakshmikantham and Leela [9].

In what follows, the symbols $\partial D, \bar{D}$ denote the strong boundary and closure of the set $D$, respectively. We denote by $B_{r}(0)$ the open ball of $X$ with center at zero and radius $r>0$. The symbol $\rightarrow(\rightarrow)$ means strong

1991 Mathematics Subject Classification. Primary 47H17; Secondary 47H05, 47H10.

Key words and phrases. Maximal monotone operator, $m$-accretive operator, compact perturbation, Leray-Schauder degree theory, cone, eigenvalue problem, compact resolvent, Kartsatos' degree theory.

Received: January 15, 1997. 
(weak) convergence. We denote by $N, R_{+}$the set of positive integers and the set $[0, \infty)$, respectively.

An operator $T: X \supset D(T) \rightarrow 2^{X}$ is called "bounded" if $T(A)=\cup\{T x:$ $x \in A\}$ is bounded for any bounded set $A \subset D(T)$. The operator $T$ is said to be " $\phi$-expansive" on $E \subset D(T)$ if there exists a continuous strictly increasing function $\phi: R_{+} \rightarrow R_{+}$such that $\phi(0)=0$ and

$$
\|u-v\| \geq \phi(\|x-y\|)
$$

for every $x, y \in E$ and all $u \in T x, v \in T y$. $T$ is called "compact" if it continuous and maps bounded subsets of $D(T)$ onto relatively compact sets. It is "demicontinuous" ("completely continuous") if it is strong-weak (weakstrong) continuous on $D(T)$. A linear operator in a reflexive Banach space is completely continuous if and only if it is compact.

By a "cone" we mean a closed and convex subset $K$ of $X$ which is closed under multiplication by nonnegative scalars and such that $K \cap(-K)=\{0\}$.

Recently, Guan and Kartsatos [4] established several results concerning the eigenvalue problem for a pair of operators $T$ and $C$, where $T$ is at least accretive or monotone while $C$ is at least compact or bounded and continuous.

One of our objectives in this paper is to complement and improve the above results by using a well known theorem of Guo and Leray-Schauder degree theory. Our second objective is to provide an affirmative answer to a problem of Kartsatos [8] concerning ranges of perturbed maximal monotone operators (Theorem 2.4).

Before we state and prove our main results, we need some auxiliary results which follow. Unless otherwise stated, the symbol $d(\cdot, \cdot, \cdot)$ denotes the LeraySchauder degree.

The following two lemmas can be found in [4].

Lemma 1.1. Let $C: \bar{D} \rightarrow X$ be compact, where $D \subset X$ is open and bounded. Assume that there exists $y_{0} \in X$ such that $y_{0} \neq 0$ and $(I-C) x \neq$ $\lambda y_{0}, \lambda \geq 0, x \in \partial D$. Then $d(I-C, D, 0)=0$.

Lemma 1.2. Let $X$ be a real infinite dimensional Banach space and $K \subset X$ be a compact set. Assume that there exists a positive constant $\alpha$ such that $\|x\| \geq \alpha$, for every $x \in K$. Then there exists $y_{0} \in X$ with $\left\|y_{0}\right\|=1$ and $\left\{t y_{0}: t \geq \alpha\right\} \cap K=\emptyset$.

We can now use the above Lemmas to deduce the following known result due to Guo [5]. Its proof is given for completeness.

Theorem 1.1. Let $X$ be a real infinite dimensional Banach space and let $D \subset X$ be open and bounded. Let $C: \bar{D} \rightarrow X$ be compact and assume that there exists a positive constant $\alpha$ such that $\|C x\| \geq \alpha, x \in \partial D$. Assume, further, that $C x=\mu x, x \in \partial D$ imply $\mu \notin(0,1]$. Then $d(I-C, D, 0)=0$.

Proof. Choose $y_{0}$ as in Lemma 1.2 with $K=\overline{-C(\partial D)}$. Then $\left\{t y_{0}: t \geq\right.$ $\alpha\} \cap K=\emptyset$. 
We shall show that there exists $\lambda^{*} \geq 1$ such that

$$
\left(I-\lambda^{*} C\right) x \neq \eta y_{0}, \quad \eta \geq 0, x \in \partial D .
$$

Indeed, assume (3) is false. Then there exist $x_{n} \in \partial D, \lambda_{n}, \eta_{n} \in(0, \infty)$ such that $\lambda_{n} \rightarrow \infty$ and

$$
\left(I-\lambda_{n} C\right) x_{n}=\eta_{n} y_{0} .
$$

Notice that $\left\{x_{n}\right\}$ is bounded. We know that $x_{n} / \lambda_{n} \rightarrow 0$ as $n \rightarrow \infty$. Hence,

$$
\left\|C x_{n}+\left(\frac{\eta_{n}}{\lambda_{n}}\right) y_{0}\right\| \rightarrow 0
$$

as $n \rightarrow \infty$. Since $\left\{C x_{n}\right\}$ is bounded, we may assume that $\eta_{n} / \lambda_{n} \rightarrow \mu$ as $n \rightarrow \infty$. Then

$$
\left\|C x_{n}+\mu y_{0}\right\| \rightarrow 0
$$

as $n \rightarrow \infty$, which implies $\mu \geq \alpha$. This contradicts the fact $\left\{t y_{0}: t \geq \alpha\right\} \cap K=$ $\emptyset$. Using Lemma 1.1, we see that $d\left(I-\lambda^{*} C, D, 0\right)=0$.

Now, we consider the following compact homotopy

$$
H(t, x) \equiv x-\left[t \lambda^{*}+(1-t)\right] C x, \quad t \in[0,1], x \in \bar{D} .
$$

By our assumption, $H(t, x) \neq 0, t \in[0,1], x \in \partial D$. By the Leray-Schauder degree theory, we have $d(I-C, D, 0)=d\left(I-\lambda^{*} C, D, 0\right)=0$. The proof of Theorem 1.1 is complete.

Theorem 1.2. Let $X$ be a real Banach space and $K \subset X$ be a cone. Let $D \subset X$ be an open, bounded set. Assume that $C: \bar{D} \rightarrow K$ is a compact operator satisfying the following conditions:

(i) $\inf \{\|C x\|: x \in \partial D\}>0$;

(ii) $C x=\mu x, x \in \partial D$ imply $\mu \notin(0,1]$.

Then $d(I-C, D, 0)=0$.

Proof. Let $M=\sup \{\|x\|: x \in \bar{D}\}$ and $d=\inf \{\|C x\|: x \in \partial D\}>0$. Letting

$$
\tau_{0}>\max \left\{\frac{2 M}{d}, 1\right\}
$$

we have $x \neq \tau_{0} C x$ for all $x \in \partial D$. We shall show that there exists $\tau>\tau_{0}$ and some $u_{0} \in X$, with $u_{0} \neq 0$, such that

$$
x-\tau C x \neq t u_{0},
$$

for any $t \geq 0, x \in \partial D$. In fact, (7) holds for $t=0$ and any $\tau>\tau_{0}, u_{0} \in$ $X$. Thus, we only need to show (7) for $t>0$. If this is not the case, then for any $u \in K$, with $\|u\|=1$, there exist $x_{n} \in \partial D, \tau_{n}>\tau_{0}, t_{n} \in(0, \infty)$ such that $\tau_{n} \rightarrow \infty$ and

$$
x_{n}-\tau_{n} C x_{n}=t_{n} u .
$$

Notice that

$$
\left\|\frac{x_{n}}{\tau_{n}}-C x_{n}\right\|=\left\|\frac{t_{n}}{\tau_{n}} u\right\|=\frac{t_{n}}{\tau_{n}}
$$


and

$$
\frac{\frac{x_{n}}{\tau_{n}}-C x_{n}}{\left\|\frac{x_{n}}{\tau_{n}}-C x_{n}\right\|}=\frac{t_{n} u}{\tau_{n}\left\|\frac{x_{n}}{\tau_{n}}-C x_{n}\right\|}=u .
$$

Since $\left\{x_{n}\right\}$ is bounded and $C$ is compact, we may assume that $C x_{n} \rightarrow y$ as $n \rightarrow \infty$. From (9) we know that $-y /\|y\|=u \in K$. On the other hand, since $C: \bar{D} \rightarrow K$, we have $y \in K$ and hence $y /\|y\|=-u \in K$. Thus, $u=0$, which is a contradiction to $\|u\|=1$. By Lemma 1.1,

$$
d(I-\tau C, D, 0)=0 .
$$

Now, we consider the compact homotopy

$$
H(t, x) \equiv x-(t \tau+1-t) C x, t \in[0,1], x \in \bar{D} .
$$

We have $H(t, x) \neq 0$, for any $t \in[0,1], x \in \partial D$. By the Leray-Schauder degree theory we have $d(I-C, D, 0)=d(I-\tau C, D, 0)=0$, which completes the proof of of Theorem 1.2.

From Theorem 1.2 we have the following two results. Since their proofs are straightforward, we omit them.

Theorem 1.3. Let $X$ be a real Banach space and $K$ a cone in $X$. Let $D \subset X$ be an open, bounded and such that $0 \in D$. Assume that $C: \bar{D} \rightarrow K$ is compact and such that $\inf \{\|C x\|: x \in \partial D\}>0$. Then there exists $(\lambda, x) \in(0, \infty) \times(K \cap \partial D)$ such that $C x=\lambda x$.

Theorem 1.4. Let $X, D, K$ be as in Theorem 1.3. Assume that $C: \partial D \cap$ $K \rightarrow K$ is compact and that there exists a constant $\alpha>0$ such that $\inf \{\|C x\|: x \in \partial D \cap K\}>\alpha$. Then the conclusion of Theorem 1.3 is true.

\section{MAIN RESULTS}

We start with a theorem which improves the corresponding result (Theorem 2.5) in Guan and Kartsatos [4]. We do not assume that $X$ and $X^{*}$ are uniformly convex. We also assume that $C$ compact, but not necessarily completely continuous. Finally, we assume only the boundedness of $T$ on the intersection of a certain ball and its domain of definition.

Theorem 2.1. Let $X$ be a real infinite dimensional Banach space and let $D \subset X$ be open and bounded. Let $T: X \supset D(T) \rightarrow 2^{X}$ be an m-accretive operator with $0 \in T(0), 0 \in D \cap D(T)$ and $D \subset \overline{D(T)}$. Assume that $T\left(\overline{B_{r}(0)} \cap D(T)\right)$ is bounded, where $r=\sup \{\|x\|: x \in \partial D\}$. Let $C$ : $\overline{D(T)} \rightarrow X$ be compact and let there exist $\alpha>0$ such that $\|C x\| \geq \alpha$, for all $x \in \partial D$. Then $(i)$ for every $c>0$ there exists $\left(\lambda_{c}, x_{c}\right)$ such that

$$
0 \in T x_{c}+c x_{c}-\lambda_{c} C x_{c} ;
$$

(ii) if, moreover, $0 \notin T(D(T) \cap \partial D)$ and $T$ is $\phi$-expansive on $D(T) \cap \partial D$, then there exists $\left(\lambda_{0}, x_{0}\right) \in(0, \infty) \times \partial D$ such that $0 \in T x_{0}-\lambda_{0} C x_{0}$. 
Proof. (i) Let $c>0$ be given. Since $0 \in D$, we know that $r>0$. Set $M=\sup \left\{\|y\|: y \in T x, x \in \bar{B}_{r}(0) \cap D(T)\right\}$ and choose

$$
\lambda^{*}>\frac{(M+c r)}{\alpha} \text {. }
$$

Since $T: D(T) \rightarrow 2^{X}$ is m-accretive and $C: \overline{D(T)} \rightarrow X$ is compact, we see that $A=(T+c I)^{-1} \lambda^{*} C: \bar{D} \rightarrow D(T)$ is compact.

Now we verify that the operator $A$ satisfies all conditions in Theorem 1.1.

(I) $\inf \{\|A x\|: x \in \partial D\}>0$. If this is not the case, there exists $\left\{x_{n}\right\} \subset \partial D$ such that $A x_{n} \rightarrow 0$ as $n \rightarrow \infty$. Let $u_{n}=A x_{n}$. Then

$$
\lambda^{*} C x_{n} \in T u_{n}+c u_{n}, \quad n \in N .
$$

Thus, there exist $v_{n} \in T u_{n}$ such that

$$
v_{n}+c u_{n}=\lambda^{*} C x_{n} .
$$

Since $u_{n} \rightarrow 0$ as $n \rightarrow \infty$, we have $u_{n} \in B_{r}(0) \cap D(T)$ for all large $n \in$ $N$. Thus, $\left\|v_{n}\right\| \leq M$ for all large $n$. From (13) we have

$$
\lambda^{*}=\frac{\left\|v_{n}+c u_{n}\right\|}{\left\|C x_{n}\right\|} \leq \frac{M+c r}{\alpha},
$$

which contradicts the choice on $\lambda^{*}$.

(II) If $A x=\mu x, x \in \partial D$, then $\mu \notin(0,1]$. If this is not the case, there exist $x_{0} \in \partial D$ and $\mu_{0} \in(0,1]$ such that $A x_{0}=\mu_{0} x_{0}$. Then $\mu_{0} x_{0} \in \overline{B_{r}(0)} \cap D(T)$ and $\lambda^{*} C x_{0} \in T\left(\mu_{0} x_{0}\right)+c \mu_{0} x_{0}$. Thus we can find $v_{0} \in T\left(\mu_{0} x_{0}\right)$ such that

$$
v_{0}+c \mu_{0} x_{0}=\lambda^{*} C x_{0} .
$$

Consequently,

$$
\lambda^{*}=\frac{\left\|v_{0}+c \mu_{0} x_{0}\right\|}{\left\|C x_{0}\right\|} \leq \frac{M+c r}{\alpha},
$$

i.e., a contradiction. By Theorem 1.1, we have $d(I-A, D, 0)=0$, i.e.,

$$
d\left(I-(T+c I)^{-1} \lambda^{*} C, D, 0\right)=0 .
$$

Now we construct a compact homotopy as follows:

$$
H(t, x) \equiv x-(T+c I)^{-1} \lambda^{*} t C x, \quad t \in[0,1], x \in \bar{D} .
$$

Indeed, for every $t \in[0,1], \quad(T+c I)^{-1} \lambda^{*} t C: \bar{D} \rightarrow D(T)$ is relatively compact. On the other hand, for every $\left\{t_{n}\right\} \subset[0,1]$, with $t_{n} \rightarrow t$ as $n \rightarrow \infty$, and all $x \in \bar{D}$, we have

$$
\begin{gathered}
\left\|(T+c I)^{-1} \lambda^{*} t_{n} C x-(T+c I)^{-1} \lambda^{*} t C x\right\| \\
\leq \frac{\lambda^{*}}{c} \sup \{\|C x\|: x \in \bar{D}\}\left|t_{n}-t\right| \rightarrow 0
\end{gathered}
$$

as $n \rightarrow \infty$. Hence, $(T+c I)^{-1} \lambda^{*} t C:[0,1] \times \bar{D} \rightarrow D(T)$ is a compact operator. Notice that $H(0, \cdot)=I, \quad 0 \in D$. We have $d(H(0, \cdot), D, 0)=1$. By the Leray-Schauder degree theory, we must have $\left(t_{c}, x_{c}\right) \in(0,1] \times \partial D$ such that $x_{c}=(T+c I)^{-1} \lambda^{*} C x_{c}$. This says that $0 \in T x_{c}+c x_{c}$, where $\lambda_{c}=\lambda^{*} t_{c} \in(0, \infty)$. 
(ii) We take $c=\frac{1}{n}$. Then there exists $\left(\lambda_{n}, x_{n}\right) \in(0, \infty) \times \partial D$ such that

$$
0 \in T x_{n}+\frac{1}{n} x_{n}-\lambda_{n} C x_{n} .
$$

Thus, $v_{n}+\frac{1}{n} x_{n}=\lambda_{n} C x_{n}$, where $v_{n} \in T x_{n}$. Since $\left\{x_{n}\right\} \subset \bar{B}_{r}(0) \cap D(T)$, we have $\left\|v_{n}\right\| \leq M$. Hence $\left\{\lambda_{n}\right\}$ must be bounded. We may assume that $\lambda_{n} \rightarrow \lambda_{0}, \quad C x_{n} \rightarrow y$ as $n \rightarrow \infty$. Then $v_{n} \rightarrow \lambda_{0} y$ as $n \rightarrow \infty$. Since $T$ is $\phi$ expansive on $D(T) \cap \partial D$, we know that $x_{n} \rightarrow x \in D(T)$ and $\lambda_{0} y \in T x$. Hence $C x_{n} \rightarrow C x$ as $n \rightarrow \infty$. So, $y=C x$. Thus, we have $\lambda_{0} C x \in T x$, i.e., $0 \in T x-\lambda_{0} C x$, where $\left(\lambda_{0}, x_{0}\right) \in(0, \infty) \times \partial D$. The proof of Theorem 2.1 is finished.

For cones of Banach spaces we have the following two theorems.

Theorem 2.2. Let $X$ be a real Banach space with a cone $K$. Let $T: X \supset$ $D(T) \rightarrow 2^{X}$ have a continuous single-valued inverse $T^{-1}: X \rightarrow D(T)$. Assume that $C: \overline{D(T)} \rightarrow K$ is compact and there exist an open bounded set $G$ and a constant $\alpha>0$ such that: $\bar{G} \subset D(T), 0 \in T(G), T(G)$ is bounded and $\|C x\| \geq \alpha, x \in \partial G$. Then there exists $(\lambda, x) \in(0, \infty) \times \partial G$ such that $0 \in \lambda T x-C x$.

Proof. Since $T^{-1}: X \rightarrow D(T)$ is continuous, we know that $T(G)$ is open and $T(\bar{G})$ is closed, moreover, $\partial T(G) \subset T(\partial G)$. Notice that $C T^{-1}: \overline{T(G)} \rightarrow K$ is compact and $\left\|C T^{-1} u\right\| \geq \alpha, u \in \partial T(G)$. By Theorem 1.3, there exists $(\lambda, u) \in(0, \infty) \times K \cap \partial T(G)$ such that $C T^{-1} u=\lambda u$. Letting $x=T^{-1} u$, we have $0 \in \lambda T x-C x, x \in \partial G$. The proof of Theorem 2.2 is finished.

Theorem 2.3. Let $X$ be a real Banach space and $K \subset X$ be a cone. Let $T: D(T) \subset K \rightarrow X$ be an m-accretive operator with $T 0=0$. Let $D$ be a bounded open in $X$ with $0 \in D(T) \cap D$ and $D \subset \overline{D(T)}$. Assume that $T\left(\overline{B_{r}(0)} \cap D(T)\right)$ is bounded, where $r=\sup \{\|x\|: x \in \partial D\}$. Let $C: \overline{D(T)} \rightarrow X$ be compact and assume that there exists $\alpha>0$ such that $\|C x\| \geq \alpha, x \in \partial D$. Then

(i) For every $c>0$, there exists $\left(\lambda_{c}, x_{c}\right) \in(0, \infty) \times \partial D \cap K$ such that $0 \in T x_{c}+c x_{c}-\lambda_{c} C x_{c}$;

(ii) if, moreover, $0 \notin T(D(T) \cap \partial D)$ and $T$ is $\phi$-expansive on $D(T) \cap \partial D$, then there exists $\left(\lambda_{0}, x_{0}\right) \in(0, \infty) \times \partial D \cap K$ such that $0 \in T x_{0}-\lambda_{0} C x_{0}$.

Proof. The proof is similar to the proof of Theorem 2.1. It is therefore omitted.

We now turn our attention to an open problem stated by Kartsatos in [8].

Let $X$ be a real reflexive Banach space with norm $\|\cdot\|$ and normalized duality mapping $J$. As it is often assumed, the spaces $X, X^{*}$ are locally uniformly convex. Thus, $J$ is a bicontinuous mapping.

An operator $T: X \supset D(T) \rightarrow 2^{X^{*}}$ is "monotone" if for every $x, y \in D(T)$ and $u^{*} \in T x, v^{*} \in T y$ we have

$$
<u^{*}-v^{*}, x-y>\geq 0
$$


A monotone operator $T$ is "maximal monotone" if $T+\lambda J$ is surjective for all $\lambda>0$. An operator $T: X \supset D(T) \rightarrow Y$, with $Y$ another real Banach space, is "bounded" if it maps bounded subsets of $D(T)$ onto bounded sets. It is "compact" if it is continuous and maps bounded subsets of $D(T)$ onto relatively compact sets.

Recently, Kartsatos [8, Theorem 7] proved the following result.

Theorem K. Let $T: X \supset D(T) \rightarrow 2^{X^{*}}$ be maximal monotone and $C$ : $D(T) \rightarrow X^{*}$. Let $(T+J)^{-1}$ be compact. Let $G \subset X$ be open, bounded and such that, for some $z \in D(T) \cap G$ and $v^{*} \in T z$,

$0 \notin T x-v^{*}, \quad<u^{*}+C x, x-z>>0, \quad\left(x, u^{*}\right) \in(D(T) \cap \partial G) \times T x$.

Assume, further, that the operator $C(\lambda T+J)^{-1}$ is compact, where $\lambda$ is a fixed constant, and the set $C(D(T) \cap \bar{G})$ is bounded. Then $0 \in(T+C)(D(T) \cap \bar{G})$.

Kartsatos asked in [8] the following question: is Theorem $\mathrm{K}$ true without the assumption that $0 \notin T x-v^{*}$, for every $x \in D(T) \cap \partial G$ ?

We shall solve the above open problem, in the affirmative, by using Kartsatos' degree theory from [7]. For this purpose, we shall first solve the perturbed problem:

$$
0 \in T x+C x+\epsilon J x .
$$

By a limiting process, we can then pass to the solution of the original problem. The key step of the proof is to construct a homotopy equation:

$$
u=H(t, u) \equiv-t C(T+\epsilon J)^{-1} u, t \in[0,1], u \in X^{*},
$$

which satisfies the condition of Kartsatos [7, Theorem 1].

Theorem 2.4. Let the assumptions of Theorem $K$ be satisfied except, possibly, the one on $T x-v^{*}$. Then $0 \in(T+C)(D(T) \cap \bar{G})$.

Proof. By the proof of Kartsatos [8, Theorem 7], we see that it suffices to show Theorem 2.4 for $z=0$ and $0 \in T 0$. Otherwise, we reduce the problem to this case by a suitable transformation.

Let $\epsilon \in(0,1)$ be given. Set $U=(T+\epsilon J)(D(T) \cap G)$, and $V=(T+$ $\epsilon J)(D(T) \cap \bar{G})$.

Since $(T+\epsilon J)^{-1}: X^{*} \rightarrow D(T)$ is continuous, we know that $U \subset X^{*}$ is open and $V \subset X^{*}$ is closed. Thus, $\bar{U} \subset V$.

Observing that $\bar{U}=U \cup \partial U$ and $V=U \cup(T+\epsilon J)(D(T) \cap \partial G)$, we have

$$
\partial U \subset(T+\epsilon J)(D(T) \cap \partial G) .
$$

We solve first the perturbed problem

$$
0 \in T x+C x+\epsilon J x .
$$

Since $T: X \supset D(T) \rightarrow 2^{X^{*}}$ is maximal monotone, we see that (19) is equivalent to

$$
u=-C(T+\epsilon J)^{-1} u .
$$

We now consider the homotopy equation

$$
u=H(t, u) \equiv-t C(T+\epsilon J)^{-1} u, \quad t \in[0,1], u \in X^{*} .
$$


It is easy to see from the resolvent identity for maximal monotone operators (see, for example, [8]) that $C(T+\epsilon J)^{-1}: X^{*} \rightarrow X^{*}$ is compact. Notice that $H(t, \cdot) \bar{U}=-t C(T+\epsilon J)^{-1} \bar{U} \subset-t C(D(T) \cap \bar{G})$ is bounded. By Kartsatos' degree theory [7, Theorem 1$]$, we only need to prove that the equation (21) has no solution in $\partial U$. Assume that the contrary is true. Then there exists $x_{t} \in \partial U$ such that

$$
x_{t}=-t C(T+\epsilon J)^{-1} x_{t} .
$$

Setting $u_{t}=(T+\epsilon J)^{-1} x_{t}$, we see that $0 \in T u_{t}+\epsilon J u_{t}+t C u_{t}$.

Noting that $x_{t} \in \partial U \subset V=(T+\epsilon J)(D(T) \cap \partial G)$, we have $u_{t}=(T+$ $\epsilon J)^{-1} x_{t} \in D(T) \cap \partial G$. By our assumption, for $u^{*} \in T u_{t}$,

$$
\begin{aligned}
0 & =<u^{*}+t C u_{t}+\epsilon J u_{t}, u_{t}> \\
& =<(1-t) u^{*}+t u^{*}+t C u_{t}, u_{t}>+\epsilon\left\|u_{t}\right\|^{2} \\
& =(1-t)<u^{*}, u_{t}>+t<u^{*}+C u_{t}, u_{t}>+\epsilon\left\|u_{t}\right\|^{2} \\
\geq & \epsilon\left\|u_{t}\right\|^{2}>0,
\end{aligned}
$$

i.e., a contradiction. Hence

$$
d\left(I+C(T+\epsilon J)^{-1}, U, 0\right)=d(I, U, 0)=1 .
$$

This shows that the equation (20) is solvable in $u \in U$, and hence the equation (19) is solvable in $x \in D(T) \cap \bar{G}$.

At this point we can choose $\epsilon_{n}=\frac{1}{n}$. Then there exists $x_{n} \in D(T) \cap \bar{G}$ such that

which leads to

$$
0 \in T x_{n}+C x_{n}+\frac{1}{n} J x_{n},
$$

$$
x_{n}=(T+J)^{-1}\left(\left(1-\frac{1}{n}\right) J x_{n}-C x_{n}\right) .
$$

Since $\left\{J x_{n}\right\},\left\{C x_{n}\right\}$ are bounded and $(T+J)^{-1}$ is compact, we see that $\left\{x_{n}\right\}$ lies in a compact set. Without loss of generality, we may assume that $x_{n} \rightarrow x$ as $n \rightarrow \infty$.

It follows from $(22)$ that

$$
C x_{n}=C(T+J)^{-1}\left(\left(1-\frac{1}{n}\right) J x_{n}-C x_{n}\right) .
$$

Since $\left\{J x_{n}\right\},\left\{C x_{n}\right\}$ are bounded and $C(T+J)^{-1}$ is compact, we see that $\left\{C x_{n}\right\}$ lies in a compact set. We may assume that $C x_{n} \rightarrow y$ as $n \rightarrow \infty$. Consequently, taking limit on both sides of (23), we obtain

$$
y=C(T+J)^{-1}(J x-y) .
$$

Set $u=(T+J)^{-1}(J x-y)$. Then $y=C u$ and $J x-y \in T u+J u$, which implies that $J x \in T u+C u+J u$.

On the other hand, by taking the limit on both sides of (22), we have

$$
x=(T+J)^{-1}(J x-y)=u,
$$

and hence $J u=J x, 0 \in T u+C u, u \in D(T) \cap \bar{G}$, which ends the proof of Theorem 2.4. 
Remark 2.1. By Theorem 2.4, we know that Corollary 4 of Kartsatos [8] is true without the assumption that $0 \notin T x$, for every $x \in D(T) \cap \partial G$.

\section{REFERENCES}

[1] V. Barbu, Nonlinear Semigroups and Differential Equations in Banach Spaces, Noordhoff Int. Publ., Leyden, the Netherlands, 1975.

[2] F. E. Browder, Nonlinear operators and nonlinear equations of evolution in Banach spaces, Proc. Sympos. Pure Math., 18, Amer. Math. Soc., Providence, 1976.

[3] Z. H. Ding and A. G. Kartsatos, Nonzero solutions of nonlinear equations involving compact perturbations of accretive operators in Banach spaces, Nonl. Analysis, 25 (1996), 1333-1342.

[4] Z. Y. Guan and A. G. Kartsatos, On the eigenvalue problem for perturbations of nonlinear accretive and monotone operators in Banach spaces, Nonl. Analysis, 27 (1996), 125-141.

[5] D. J. Guo, Nonlinear Functional Analysis, Shandong Press, Shandong, China, 1985.

[6] A. G. Kartsatos, Recent results involving compact perturbations and compact resolvents of accretive operators in Banach spaces, Proc. First World Congress Nonl. Analysts, Tampa, Florida, 1992, Walter De Gruyter, New York, 1995, pp. 2197-2222.

[7] A. G. Kartsatos, On the perturbation theory of $m$-accretive operators in Banach spaces, Proc. Amer. Math. Soc. 124 (1996), 1811-1820.

[8] A. G. Kartsatos, New results in the perturbation theory of maximal monotone and $m$-accretive operators in Banach spaces, Trans. Amer. Math. Soc. 348 (1996), 16631707.

[9] V. Lakshmikantham and S. Leela, Nonlinear Differential equations in Abstract Spaces, Pergamon Press, Oxford, 1981.

[10] S. P. Wu, Functional Analysis and Its Applications, Zhejiang Univ. Press, Zhejiang, China, 1990.

Department of Mathematics

Shijiazhuang Mechanical Engineering College

ShiJIAZHuAng, 050003 PEOPLE'S REPUBLIC OF CHINA

E-mail address: hzhou@chuma.cas.usf.edu

Department of Mathematics

University of South Florida

TAMPA, FL 33620-5700, USA

E-mail address: hermes@math.usf.edu 


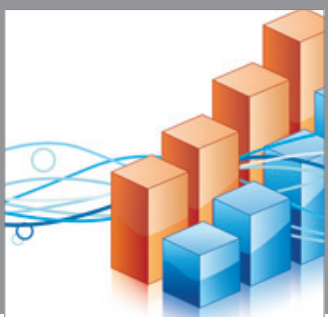

Advances in

Operations Research

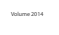

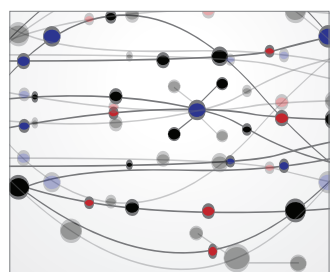

\section{The Scientific} World Journal
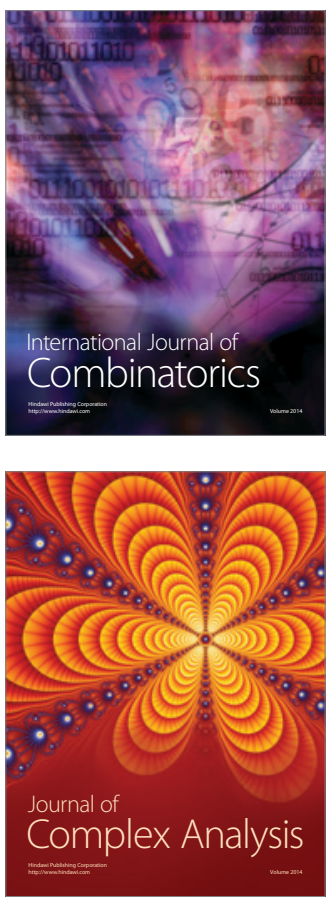

International Journal of

Mathematics and

Mathematical

Sciences
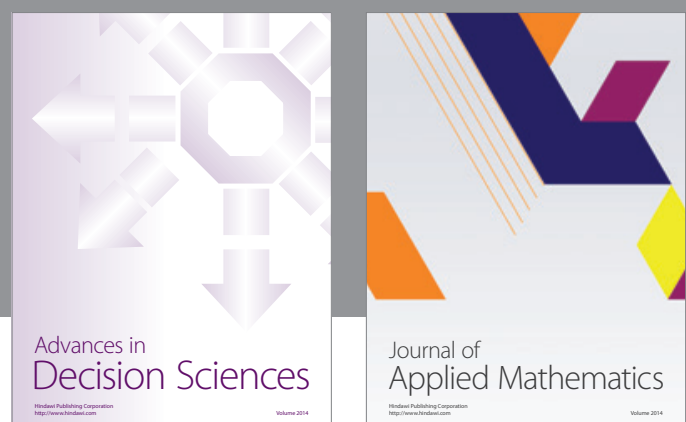

Journal of

Applied Mathematics
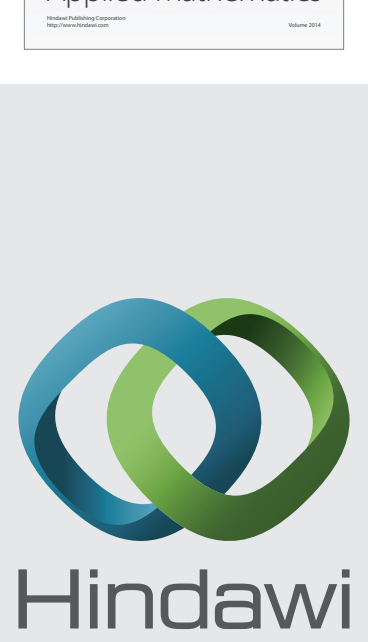

Submit your manuscripts at http://www.hindawi.com
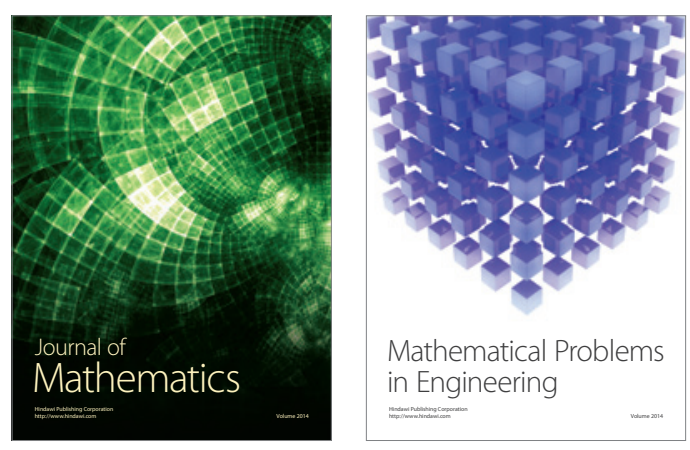

Mathematical Problems in Engineering
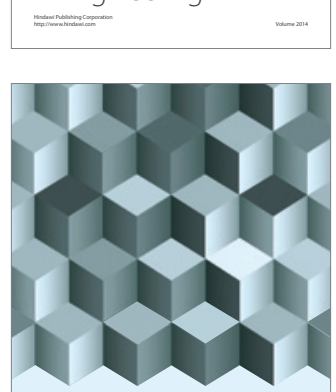

Journal of

Function Spaces
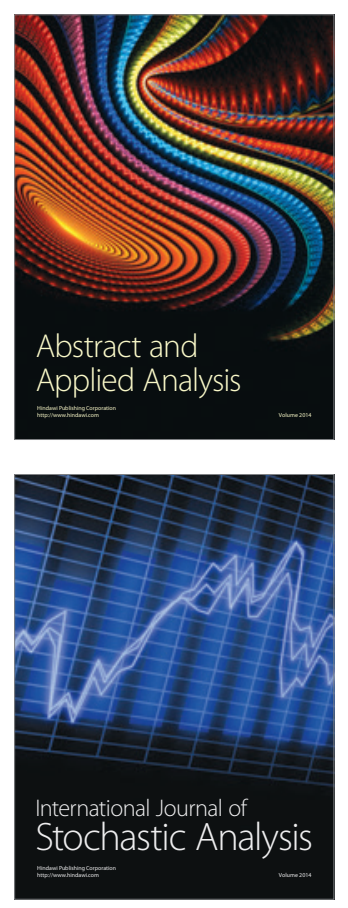

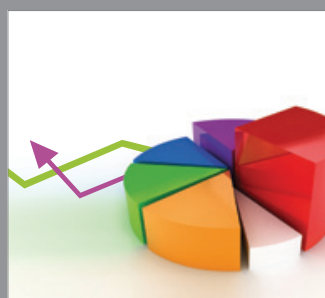

ournal of

Probability and Statistics

Promensencen
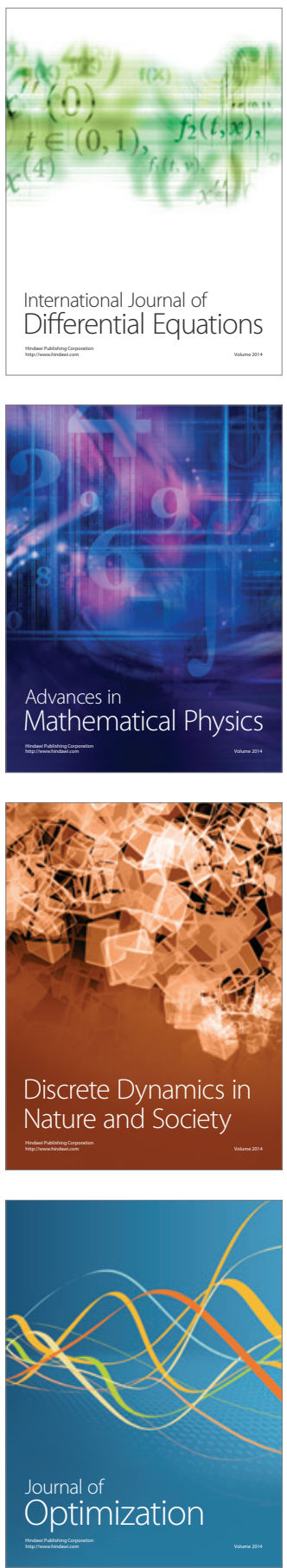